\title{
PERCEPÇÃO DE USUÁRIOS SOBRE A ASSISTÊNCIA PRESTADA NA SAÚDE PÚBLICA
}

\author{
Vanessa Cruz Santos ${ }^{1}$, Karla Ferraz dos Anjos ${ }^{1}$, Ceres Neide Almeida Costa ${ }^{2}$
}

RESUMO: Esta pesquisa teve por objetivos conhecer a percepção de moradores de Vitória da Conquista, Estado da Bahia, sobre a assistência prestada na saúde pública, e identificar problemas recorrentes do Sistema Único de Saúde naquele contexto. Trata-se de um estudo exploratório e quantitativo, realizado em janeiro de 2010 com 100 usuários. A coleta de dados se deu por meio da aplicação de questionário estruturado e analisado com auxílio de estatística descritiva. Os resultados apontaram que parte dos entrevistados desconhece algum dos vários serviços prestados na atenção primária de saúde, além de estar insatisfeitos com o funcionamento do Sistema Único de Saúde oferecido.

PALAVRAS-CHAVE: Participação comunitária; Sistema único de saúde; Atenção primária à saúde; Saúde pública.

\section{USERS' PERCEPTIONS OF ASSISTANCE GIVEN BY THE PUBLIC HEALTH SYSTEM}

ABSTRACT: This research's aims were two-fold: to reveal how assistance given by the Public Health Service was perceived among residents of Vitória da Conquista, in the state of Bahía, and to identify recurrent problems in the Single Health System (free-of-charge Public Health system provided by the Brazilian government) in that context. It is an exploratory and quantitative study, carried out in January 2010 with 100 health-service users. Data collection was carried out via structured questionnaires and was analysed with the help of descriptive statistics. The results indicate that some of the interviewees are unaware of various services provided by primary health care, apart from being dissatisfied with how the Single Health System works.

KEYWORDS: Community participation; Single health system; Primary health care; public health.

\section{PERCEPCIÓN DE USUARIOS SOBRE LA ASISTENCIA PRESTADA EN LA SALUD PÚBLICA}

RESUMEN: Esta investigación tuvo por objetivos conocer la percepción de personas que viven en Vitória da Conquista, estado de Bahia, sobre la asistencia prestada en la salud pública, así como identificar problemas recurrentes del Sistema Único de Salud en aquel contexto. Es un estudio exploratorio y cuantitativo, realizado en enero de 2010 con 100 usuarios. Los datos fueron recogidos por medio de aplicación de cuestionario estructurado y analizados con ayuda de estadística descriptiva. Los resultados apuntaron que parte de los entrevistados desconoce algunos de los varios servicios prestados en la atención primaria de salud, además de estar insatisfechos con el funcionamiento del Sistema Único de Salud ofrecido. PALABRAS CLAVE: Participación de la comunidad; Sistema único de salud; Atención primaria a la salud; Salud pública.

${ }^{1}$ Enfermeira Assistente da Clínica de Oncologia Médica - ONCOMED. Especialista em Saúde Coletiva com ênfase em PSF. ${ }^{2}$ Enfermeira. Especialista em Auditoria de Sistemas de Saúde. Professora da Faculdade de Tecnologia e Ciências - FTC.

\author{
Autor correspondente: \\ Vanessa Cruz Santos \\ Clínica de Oncologia Médica - ONCOMED \\ Caminho 29, 11 - 45000-000 - Vitória da Conquista-BA-Brasil \\ E-mail: autoraautoria@hotmail.com
}

Recebido: 08/08/10 Aprovado: 07/01/11 


\section{INTRODUÇÃO}

No Brasil, a Saúde Pública é muito discutida na atualidade e, para entender as queixas emergentes, é preciso compreender como os serviços de saúde coletiva podem e devem oferecer atendimento aos usuários.

Conforme a Lei 8.080, art. 7o, as ações e serviços públicos de saúde e os serviços privados contratados ou conveniados que integram o Sistema Único de Saúde (SUS) devem ser desenvolvidos de acordo com as diretrizes previstas no artigo 198 da Constituição Federal, obedecendo, dentre outros, os princípios de: universalidade de acesso; integralidade de assistência, entendida como um conjunto articulado e contínuo das ações e serviços preventivos e curativos, individuais e coletivos; igualdade da assistência à saúde, sem preconceitos ou privilégios de qualquer espécie; e participação da comunidade ${ }^{(1)}$.

A Saúde Pública é uma ciência e arte que trabalha no intuito de evitar doenças, prolongar a vida e desenvolver a saúde física e mental. Ela busca, por meio de esforços organizados da comunidade, o saneamento do meio ambiente, o controle de infecções, a organização de serviços de saúde para o diagnóstico precoce e o tratamento preventivo de doenças ${ }^{(2)}$.

Os profissionais de saúde devem exercer suas funções com responsabilidade e competência para prestar assistência de qualidade aos usuários que utilizam os serviços públicos de saúde. Consideramos que, na atenção primária, é preciso maior atuação dos profissionais de saúde, uma vez que a ênfase do cuidar está voltada à promoção e prevenção de agravos a saúde. No entanto, deve-se mencionar também o papel dos usuários deste sistema. Afinal, eles fazem parte da sua construção.

Esta pesquisa teve como objetivos conhecer a percepção de moradores de Vitória da Conquista/BA sobre a assistência prestada na saúde pública, bem como identificar problemas recorrentes do SUS local.

\section{METODOLOGIA}

Trata-se de um estudo de abordagem exploratória, quantitativa. Exploratório, pois proporciona maior familiaridade com o problema, com vistas à construção de hipóteses. Neste tipo de estudo, objetiva-se o aprimoramento de ideias ou a descoberta de intuições. Seu planejamento é, portanto, bastante flexível, de modo que possibilite a consideração dos mais variados aspectos relativos ao fato estudado ${ }^{(3)}$. É quantitativo, por considerar que tudo pode ser quantificável, entendendo, assim, que se pode traduzir em números opiniões e informações para classificá-las e analisálas ${ }^{(4)}$.

O estudo foi realizado com 100 moradores com idade igual ou superior a 18 anos de idade, do bairro centro do Município de Vitória da Conquista, que responderam a um questionário estruturado aplicado pelos pequisadores no período de 8 a 14 de janeiro de 2010, nos turnos matutinos e vespertinos. O instrumento de coleta de dados foi construído especialmente para o estudo e conforme seus objetivos, buscando, assim, propiciar a coleta de informações sobre os serviços de saúde pública, em especial da atenção primária do município.

A Cidade de Vitória da Conquista está localizada na região sudoeste do Estado da Bahia, com população aproximada de 308.204 habitantes e área urbana de $204 \mathrm{~km}^{2}$. Sua principal atividade econômica, fator determinante da economia na zona urbana, está no comércio e na prestação de serviços; já no setor rural, a cafeicultura, horticultura, olericultura e fruticultura, além das culturas de bovinos, caprinos e suínos são as principais atividades econômicas ${ }^{(5)}$.

A amostragem foi do tipo não probabilística por conveniência, uma vez que possibilita realizar a seleção dos elementos da população para compor a amostra dependendo, ao menos em parte, do julgamento do pesquisador ou do entrevistador no campo. Os mesmos selecionam membros da população mais acessíveis no momento da aplicação do método da investigação ${ }^{(6)}$. Os resultados foram organizados e analisados por meio de estatística descritiva.

O estudo foi realizado de acordo a resolução $n$. 196/96 do Conselho Nacional de Saúde, após aprovação do Comitê de Ética em Pesquisa da Universidade Estadual do Sudoeste da Bahia, sob o número 133/2009.

\section{RESULTADOS}

Os participantes deste estudo caracterizam-se, em sua maioria, por serem jovens adultos, $57 \%$ de indivíduos com faixa etária de 18 a 25 anos de idade; $29 \%$ têm entre 26 a 36 anos; e 14\% são maiores de 34 anos. A maioria é do sexo feminino, totalizando $72 \%$. De acordo o grau de escolaridade, $49 \%$ tinham ensino médio completo, $21 \%$ ensino médio incompleto, $15 \%$ superior incompleto, $12 \%$ ensino fundamental incom- 
pleto e 3\% superior completo. Quanto à renda individual, $74 \%$ recebiam até 1 salário mínimo mensal, 19\% entre 2 e 4 salários mínimos, 3\% mais de 4 salários mínimos e $4 \%$ dos entrevistados não responderam a esta questão.

Quando se questionou aos entrevistados quanto a ser ou não usuários do SUS, $28 \%$ responderam que não são e $72 \%$ responderam ser usuários do sistema. Com relação aos serviços de saúde pública prestados na atenção primária, 29\% dos participantes disseram conhecer algum dos serviços oferecidos, 39\% desconhecem alguns desses serviços e 32\% não responderam à pergunta.

Em relação ao grau de satisfação, $44 \%$ referiram não estar satisfeitos com a prestação dos serviços públicos de saúde; $28 \%$ não responderam; $24 \%$ referiram estar parcialmente satisfeitos; e apenas $4 \%$ responderam estar satisfeitos.

Conforme uma classificação avaliativa referindo o atendimento prestado, $44 \%$ o julgaram como "regular", 28\% não responderam, 20\% classificaram como "péssimo", $7 \%$ como "bom" e $1 \%$ referiram que o atendimento é "ótimo". Ainda, 71\% dos usuários referiram que se aborrecem quando procuram atendimento pelo SUS, 28\% não responderam a questão e apenas $1 \%$ afirmaram não ter aborrecimentos com $\mathrm{o}$ atendimento neste sistema.

Quando questionados sobre os atendimentos oferecidos pela Estratégia Saúde da Família (ESF), $35 \%$ referiram acreditar que os programas trabalham em parte com a atenção primária, $18 \%$ disseram que não, $17 \%$ afirmaram que sim e $30 \%$ não responderam a questão.

Em relação ao registro de reclamação oficial ao setor de ouvidoria do SUS, apenas 5\% afirmaram já ter realizado; $67 \%$ nunca reclamaram e $28 \%$ não responderam a pergunta. Entre os respondentes, $67 \%$ afirmaram nunca ter participado de assembleia ou conferência municipal de saúde, $28 \%$ não responderam a questão e apenas $5 \%$ afirmaram já ter participado.

Ao serem questionados se já procuraram informações sobre os recursos disponíveis para a atenção primária à saúde da população, $66 \%$ responderam que nunca procuraram informações a respeito, 33\% disseram que sim e $1 \%$ não respondeu.

\section{DISCUSSÃO}

A Constituição Federal diz, no artigo 196, que a saúde é direito de todos e dever do Estado, garantido mediante políticas sociais e econômicas que visem à redução do risco de doença e de outros agravos e ao acesso universal e igualitário às ações e serviços para sua promoção, proteção e recuperação ${ }^{(1)}$.

Resultados de pesquisa nacional realizada pelo Instituto Brasileiro de de Geografia e Estatística, em 2003 , revela que $24,5 \%$ da população brasileira possuem cobertura de plano de saúde, enquanto $75,4 \%$ utilizam o SUS ${ }^{(7)}$. Entretanto, se for considerado que este sistema é responsável pela vigilância de produtos e serviços e, ainda, pela vigilância epidemiológica, pode-se dizer que $100 \%$ dos brasileiros são atendidos de forma direta ou indireta pelo SUS. Este Sistema não está direcionado apenas para o atendimento direto prestado pelas equipes de saúde, mas também por meio da atuação das vigilâncias à saúde e outros serviços que são realizados em prol da sociedade.

Os cuidados primários de saúde são baseados em métodos e tecnologias práticas, cientificamente bem fundamentados, colocados ao alcance universal de indivíduos e famílias da comunidade. Eles representam o primeiro nível de contato dos indivíduos e da família com o sistema de saúde, refletindo nas condições econômicas, características socioculturais e políticas do país e de suas comunidades. Os cuidados primários se baseiam na aplicação dos resultados relevantes da pesquisa social, biomédica e de serviços de saúde e da experiência em saúde pública, proporcionando serviços de proteção, cura e reabilitação, conforme as necessidades de cada indivíduo, devendo se preocupar com a nutrição apropriada, previsão adequada de água de boa qualidade e saneamento básico, cuidados de saúde materno-infantil, planejamento familiar, imunização contra doenças infecciosas, prevenção e controle de doenças localmente endêmicas, tratamento de doenças, lesões e fornecimento de medicamentos ${ }^{(8)}$.

O maior percentual dos entrevistados desconhece algum serviço da atenção primária à saúde, antes denominada "básica" e que dava a falsa ideia de "cesta básica", um serviço oferecido para suprir as necessidades mais evidentes do indivíduo. Essa ideia é incorreta, uma vez que a atenção primária vai além deste viés e não significa apenas um setor da saúde pública, pois está entrelaçada com os níveis de atenção secundária e terciária.

O SUS é um sistema amplo que depende de uma cadeia processual. Logo, a prestação de seus serviços é complexa. Conforme a pesquisa, diversos conquistenses não estão satisfeitos com os serviços de 
saúde pública prestado em seu município. De acordo com as leis e diretrizes que regem esse sistema, este deveria ser o mais completo serviço de saúde oferecido à população brasileira. No entanto, algo está em desequilíbrio, pois é grande o número de usuários que reclamam do que lhe é oferecido nos cuidados à saúde.

Poucos são os indivíduos que conceituam como "bom" ou "ótimo" o atendimento prestado pela equipe de saúde. O quadro de funcionários trata os usuários do SUS como indivíduos menos favorecidos, com uma ideologia de que vários usuários vêm aos serviços "pedir" e não buscar por atendimento de saúde que lhes é de direito ter. É preciso que vários destes profissionais sejam capacitados a reconhecer a essência do que são seres humanos e aprender a lidar com as diferenças, sem ter que tratá-los como "pobres".

O termo "humanização dos serviços de saúde" parece, inicialmente, algo redundante, pois tanto os executores do trabalho como os usuários são seres humanos por natureza. Contudo, o SUS passa por dificuldades e isto torna o atendimento desumano para muitos ${ }^{(9)}$.

O modelo tecnicista-hospitalocêntrico não atende as mudanças do mundo moderno e, consequentemente, às necessidades de saúde da população. Assim, as equipes da ESF apresentam novas alternativas de trabalhar a saúde, tendo a família como centro de atenção e não somente o indivíduo doente ${ }^{(10)}$. De acordo com a minoria dos participantes da pesquisa, as Equipes de Sáude da Família trabalham com atenção primária, o que se torna uma problemática, pois este modelo de atenção deve atuar de forma horizontal e estar entrelaçado em forma de redes. Além do mais, se esse nível de atenção à saúde funcionasse de acordo com os objetivos propostos, muitos gastos poderiam ser minimizados nos outros níveis de atenção.

Para que ocorram mudanças na proposta do modelo assistencial vigente, é preciso incorporar os pressupostos da Educação Permanente em Saúde, para que os profissionais atuantes nas Equipes de Saúde da Família busquem discutir e analisar a realidade do seu território e identificar as necessidades individuais e coletivas, construindo com profissionais e a sociedade uma nova forma de agir na saúde e para a saúde ${ }^{(11)}$.

O papel do usuário do SUS como protagonista tem impacto direto na melhoria da relação entre ele e o serviço, fazendo repensar as práticas profissionais ou intervir sobre a forma de organização dos serviços, visando seu aperfeiçoamento ${ }^{(12)}$.
O Município de Vitória da Conquista conta com o serviço de ouvidoria disponível no site da Prefeitura Municipal, e todo e qualquer cidadão tem direito a opinar ou reclamar oficialmente sobre os serviços de Saúde Pública. Esse aspecto é relevante, pois, por meio do controle social, pode-se propor novas políticas públicas que sejam fundamentais para suprir as necessidades de saúde mais evidentes desta população.

As assembleias ou conferências de saúde são abertas à população, a qual faz parte do SUS e deve estar informada sobre a gestão da saúde pública de sua cidade, bem como outras informações pertinentes ao interesse dos indivíduos. É neste espaço que os cidadãos podem contribuir com suas opiniões e fazer parte do SUS, não só como usuários, mas também como parceiros dos gestores. Afinal, é dos impostos pagos por eles que são disponibilizados os recursos para a área da saúde.

A administração do SUS é, essencialmente, participativa e democrática, na qual os conselhos são responsáveis pela formulação e execução das políticas de saúde que deverão ser implantadas pelos gestores. Por isso, a fim de que os conselheiros de saúde possam, de forma consciente e responsável, deliberar por meio das políticas como combater as desigualdades sanitárias e promover a saúde da população, é necessário, que eles tenham acesso às informações sobre a realidade epidemiológica do município e seus modelos assistenciais $^{(13)}$.

Os cidadãos devem manter-se informados sobre o quanto de recursos esta disponível para a saúde pública do seu município, bem como para os serviços de atenção primária. Em outras palavras, a participação do indivíduo é fundamental para que o sistema seja cada vez mais aperfeiçoado. Logo, a sociedade deve ter conhecimento de seus direitos e deveres, pois o SUS é para todos e cada um pode contribuir de forma direta ou indireta para que ocorra uma melhor gestão dos recursos utilizados no mesmo.

Um recurso relevante é a transferência financeira Fundo a Fundo, que consiste no repasse de valores de forma regular, diretamente do Fundo Nacional de Saúde, de maneira descentralizada, para os Estados, Municípios e Distrito Federal, independentemente de convênio ou instrumento similar, destinando-se ao financiamento das ações estratégicas e serviços de saúde pública de cada esfera ${ }^{(14)}$.

Quando se define o que é SUS, segundo as leis orgânicas, pode parecer algo paradoxal com o que se vê na prática. Quantas vezes uma internação indicada 
não pode ser feita ou um medicamento prescrito não é disponibilizado pelo sistema? Por que tantas mulheres ainda morrem durante o parto? Será que o SUS é apenas uma bela ideia que ainda não se concretizou?

Ninguém pode negar que ainda há muitos problemas a serem resolvidos. Entretanto, já não ouvimos falar de lugares em que o SUS funciona bem? Provavelmente, isto se deve ao fato de ali existir dirigentes que se esforçam mais para melhorar o atendimento. Mas só isso não basta: é necessário que haja mobilização e participação coletiva e individual, pois mesmo que este sistema tenha falhas, não há melhor "plano de saúde". Afinal, que outro "plano de saúde" oferece à população ações de saúde, como o combate às doenças infecciosas, medicamento gratuito, campanhas de vacinação, ações de vigilância sanitária e atendimentos de pequena, média e alta complexidade ${ }^{(15)}$ ?

\section{CONSIDERAÇÕES FINAIS}

O SUS não é um sistema pronto, trata-se de algo mutável e processual. Portanto, mesmo que atualmente ele seja visto por vários de seus usuários como "falido", há a possibilidade de ser reformulado, reerguido e, mesmo não alcançado a perfeição, pode oferecer uma prestação de serviços de qualidade, desde que suas normas e diretrizes sejam utilizadas na prática cotidiana. Porém, é preciso a participação de todos, desde os gestores aos usuários do serviço.

Existem diversos problemas nos serviços de Saúde Pública brasileiros, entre eles a demanda maior que a oferta, o déficit de humanização no atendimento prestado pelos funcionários, bem como o de informações que são de suma importância para os indivíduos atendidos nos programas mantidos pelo sistema. A pesquisa aponta que os participantes desconhecem alguns dos serviços prestados na atenção primária.

Na realidade do Município de Vitória da Conquista, poucos foram os participantes deste estudo que estão satisfeitos com a prestação dos serviços de Saúde Pública prestados em seu município. Além disso, a maioria deles classificou o atendimento dos funcionários e equipe de saúde como "péssimo" e "regular", sendo que o número de participantes que se chateia em procurar esse tipo de serviço no município é expressivo.

Não basta cruzar os braços mediante a crise que o SUS atravessa no momento. Afinal, a saúde é direito de todos. Portanto, cada cidadão deve lutar pelos seus direitos para uma assistência de qualidade. E, apesar das falhas e contraposições, a utopia de mudança pode transformar a realidade, e este paradoxo entre teoria e prática pode ter uma solução verdadeiramente eficaz.

\section{REFERÊNCIAS}

1. Brasil. Constituição da República Federativa do Brasil. Brasília: Senado; 1998.

2. Rouquayrol MZ, Almeida Filho N. Epidemiologia e saúde. $6^{\mathrm{a}}$ ed. Rio de Janeiro: Médica e Científica; 2003.

3. Gil AC. Como elaborar projetos de pesquisa. $4^{\mathrm{a}}$ ed. São Paulo: Atlas; 2007.

4. Silva EL, Menezes EM. Metodologia da pesquisa e elaboração de dissertação. [Internet]. Florianópolis: Laboratório de Ensino a Distância da UFSC; 2001 [acesso em 10 jan 2009] Disponível: http:// projetos.inf.ufsc.br/arquivos/Metodologia $\% 20 \mathrm{da} \% 20$ Pesquisa\%203a\%20edicao.pdf

5. Prefeitura Municipal de Vitória da Conquista. [Internet]. Vitória da Conquista (BA); [acesso em 10 jan 2010]. Informações gerais sobre o município. Disponível: http://www.pmvc.com.br/v1/pmvc. php?pg=content\&id $=15$

6. Mattar FN. Pesquisa de marketing. $6^{\mathrm{a}}$ ed. São Paulo: Atlas; 2005.

7. Sousa MF, Merchán-Hamann E. Saúde da família no Brasil: estratégia de superação da desigualdade na saúde? Physis. [Internet] 2009;19(3) [acesso em 9 jan 2009]. Disponível: http://www.scielo.br/pdf/ physis/v19n3/a09v19n3.pdf

8. Organização Pan-Americana de Saúde. Conferência Internacional sobre Cuidados Primários de Saúde. Declaração de Alma-Ata. 1978. [acesso em 9 jun 2009]. Disponível: http://www.opas.org.br/coletiva/ uploadArq/Alma-Ata.pdf.

9. Ministério da Saúde (BR). Secretaria-Executiva. Núcleo Técnico de Política Naciona de Humanização. HumanizaSUS. Política Nacional de Humanização: a humanização como eixo norteador das práticas de atenção e gestão em todas as instâncias do SUS. 2004 [acesso em 19 jan 2009]. Disponível: http://bvsms. saude.gov.br/bvs/publicacoes/humanizasus_2004. pdf 
10. Rosa WAG, Labate RCL. Programa saúde da família: a construção de um novo modelo de assistência. Rev Latino-Am Enfermagem. [Internet] 2005;13(6) [ acesso em 22 mar 2009]. Disponível: http://www.scielo.br/ pdf/rlae/v13n6/v13n6a16.pdf

11. Sanes MS, Arrieche TA, Cestari MEC. A educação no discurso de uma equipe de saúde da família. Cogitare Enferm. [Internet] 2010;15(3) [acesso em 10 outubro 2010]. Disponível: http://ojs.c3sl.ufpr.br/ojs2/index. php/cogitare/article/view/18891/12200

12. Ramos DD, Lima MADS. Acesso e acolhimento aos usuários em uma unidade de saúde de Porto Alegre, Rio Grande do Sul, Brasil. Cad Saúde Pública. [Internet] 2003;19(1) [acesso em 11 jan 2010]. Disponível: http:// www.scielosp.org/pdf/csp/v19n1/14902.pdf

13. Bispo Júnior JP, Gesteira IAL. A ciência da informação e o controle social na área de saúde. 2003. [acesso em 26 maio 2009]. Disponível: http://www.cinform.ufba. br/v_anais/artigos/josebispojunior.html

14. Ministério da Saúde (BR). Fundo Nacional de Saúde: transferência fundo a fundo. 2009. [acesso em 26 dez 2009]. Disponível: http://www.fns.saude.gov.br/ Consultafundoafundo.asp

15. Stralen CJV, Ribeiro MAA. O Sistema Único de Saúde. [Internet] 2009. [acesso em 18 mar 2009]. Disponível: http://www.fozdoiguacu.pr.gov.br/noticias/link58.htm 